\title{
Potential of Environmental Enrichment to Prevent Transgenerational Effects of Paternal Trauma
}

\begin{abstract}
(20)
Katharina Gapp 1,3, Johannes Bohacek', Jonas Grossmann², Andrea M Brunner',4, Francesca Manuella', Paolo Nanni ${ }^{2}$ and Isabelle M Mansuy*,

'Laboratory of Neuroepigenetics, University of Zurich and ETH Zurich, Brain Research Institute, Neuroscience Center Zürich, Zurich, Switzerland;

${ }^{2}$ Functional Genomics Center Zurich (FGCZ), University Zurich/ETH Zurich, Zurich Switzerland

Adverse experiences in early life are risk factors for the development of behavioral and physiological symptoms that can lead to psychiatric and cognitive disorders later in life. Some of these symptoms can be transmitted to the offspring, in some cases by non-genomic mechanisms involving germ cells. Using a mouse model of unpredictable maternal separation and maternal stress, we show that postnatal trauma alters coping behaviors in adverse conditions in exposed males when adult and in their adult male progeny. The behavioral changes are accompanied by increased glucocorticoid receptor $(G R)$ expression and decreased DNA methylation of the GR promoter in the hippocampus. DNA methylation is also decreased in sperm cells of exposed males when adult. Transgenerational transmission of behavioral symptoms is prevented by paternal environmental enrichment, an effect associated with the reversal of alterations in GR gene expression and DNA methylation in the hippocampus of the male offspring. These findings highlight the influence of both negative and positive environmental factors on behavior across generations and the plasticity of the epigenome across life. Neuropsychopharmacology (2016) 4I, 2749-2758; doi:I0. I038/npp.20 I6.87; published online 6 July 2016
\end{abstract}

\section{INTRODUCTION}

Exposure to traumatic and stressful events in early life can have physiological and behavioral consequences that persist across the lifespan. Such experiences often increase the risk to develop psychiatric and cognitive disorders in adulthood (Klengel and Binder, 2015). Further, their effects can be transmitted to subsequent generations and affect the offspring similarly to the ancestors in the absence of any trauma or stress exposure (Bohacek and Mansuy, 2015; Metz et al, 2015). In some cases, however, negative experiences can have some benefits and lead to better adapted physiological and behavioral responses. Resilience is a form of adaptive response manifested by active or passive coping in adverse conditions (Franklin et al, 2012) that can follow previous trauma exposure. For instance, social avoidance is viewed as an active coping behavior that can be manifested by an adult individual after being exposed to traumatic stress in early

\footnotetext{
*Correspondence: Professor IM Mansuy, Brain Research Institute, University Zurich/Swiss Federal Institute of Technology, Winterthurerstrasse 190, Zurich 8057, Switzerland, Tel: +4I 4463533 60, Fax: +4I 4463533 03, E-mail: mansuy@hifo.uzh.ch

${ }^{3}$ Current address: Gurdon Institute, University of Cambridge, Tennis Court Road, Cambridge CB2 I QN, UK.

${ }^{4}$ Current address: Biomolecular Mass Spectrometry and Proteomics, Bijvoet Center for Biomolecular Research and Utrecht Institute for Pharmaceutical Sciences, University of Utrecht, Padualaan 8, 3584CH Utrecht, The Netherlands.

Received I5 February 2016; revised 9 May 2016; accepted 28 May 2016; accepted article preview online 9 June 2016
}

postnatal life (Franklin et al, 2011) or chronic social defeat in adulthood (Kovalenko et al, 2014). In the case of early traumatic stress, social avoidance has been shown to be accompanied by altered behavioral flexibility, and both are transmitted to the progeny (Franklin et al, 2011; Gapp et al, 2014b). Further, some maladaptive behaviors resulting from early trauma can be modulated by the environment; in particular, they can be reversed by conditions such as environmental enrichment (EE) (Leshem and Schulkin, 2012; Schloesser et al, 2010). This suggests that the effects of early life trauma, whether detrimental or beneficial, and their transmission might be counteracted by environmental factors later in life.

Mechanistically, the consequences of early exposure to trauma are complex, and implicate a combination of signaling pathways that engage the hypothalamic-pituitary-adrenal (HPA) axis. In this axis, the glucocorticoid receptor (GR) is a major component of stress responses needed for the rapid shut down of the stress response (de Kloet et al, 2005a) and for long-term adaptive processes after chronic stress (Jankord and Herman, 2008; Liu et al, 1997; Plotsky and Meaney, 1993), especially in the context of active avoidance behavior (Patacchioli et al, 1990). GR gene is subjected to epigenetic regulation (Weaver et al, 2004), and is particularly responsive to environmental factors in early life (Mueller and Bale, 2008).

Here, we used a mouse model of unpredictable maternal separation combined with unpredictable maternal stress (MSUS) to examine the consequences of traumatic stress on 
coping behaviors in adulthood and across generations, and the potential contribution of GR. We show that MSUS affects avoidance behaviors and learning in aversive environments in exposed fathers and their male offspring. This is associated with an increase in GR expression in the hippocampus, and with decreased DNA methylation of GR promoter in the hippocampus and in germ cells. We show that transmission of the effects of paternal trauma can be prevented by paternal EE, suggesting a reversibility of these effects.

\section{MATERIALS AND METHODS}

\section{Animals}

C57Bl/6J male and female mice were maintained under a reverse light-dark cycle in a temperature- and humiditycontrolled facility. Three to five mice per cage were housed with water and food ad libitum and cages were cleaned once a week. All experimental manipulations were performed during the animals' active cycle and in accordance with guidelines and regulations of the cantonal veterinary office Zurich under license 55/2012.

\section{MSUS Treatment and Breeding}

Unpredictable MSUS was conducted as previously described (Franklin et al, 2010). Briefly, adult males and females (F0) were mated, males were removed after a few days and females were maintained alone during gestation until delivery. Newborn pups (F1) were separated from their mother unpredictably (any time during the dark cycle) daily for $3 \mathrm{~h}$. During separation, dams were exposed to an additional stress consisting of either 20-min restraint in a Plexiglas tube or 5-min forced swim in cold water $\left(18^{\circ} \mathrm{C}\right)$ applied randomly any time during the $3 \mathrm{~h}$ of separation. MSUS was conducted from PND1 to PND14. Dams and pups were then left undisturbed from PND15 until weaning at PND21. About 20-30 females were bred for each MSUS experiment and only dams giving birth less than a week apart of each other were used to obtain F1 pups. Litters with less than four pups were excluded. When adult, F1 control and MSUS males were bred with naive C57Bl/6J females to generate an offspring (F2).

\section{Behavioral Testing}

The experimenter was blind to treatment for all behavioral testing and tracking was performed manually and automatically (Viewpoint System, France). Testing started with the least aversive task (light-dark box) when animals were at least 3 months old. All behavioral tests were carried out in males to avoid confounds related to the estrus cycle in females.

\section{Light-Dark Box}

Mice were placed in the lit compartment of a plastic box $(40 \times 42 \times 26 \mathrm{~cm})$ separated from a dark compartment by a divider. The lit (aversive) compartment is large $(2 / 3$ of the box), has white walls, and is brightly lit by an overhead lightbulb. The neighboring dark (safe) compartment is small ( $1 / 3$ of the box), has black walls, and is covered by a black lid. An opening in the divider $(5 \times 5 \mathrm{~cm})$ allows the animal to escape from the lit compartment to the dark compartment. Each animal was tested for $10 \mathrm{~min}$ and the time spent in each compartment and the latency to enter the dark compartment were measured manually. Light dark box data from the standard housed groups were reported before in Gapp et al (2014a).

\section{Active Avoidance Task}

Four identical operant conditioning chambers $(15.9 \times 16.5$ $\times 17.5 \mathrm{~cm}$ ) with stainless steel grid floors (TSE Systems, Germany) were placed in sound-insulated boxes. Each chamber is equipped with a shock grid floor, a nose-poke response unit ( $2 \mathrm{~cm}$ diameter) fitted with a photocell sensor, and a yellow cue light in a hole. An additional green cue light is located on the wall on top of the nose-poke module. A house light $(2.8 \mathrm{~W})$ is placed on the ceiling, and is turned on during each testing session. The nose-poke module is located on the left part of one wall, which also had a photocell sensor. Each mouse was habituated to the chamber one day for $30 \mathrm{~min}$ with the nose-poke module closed. After habituation, animals were tested for active avoidance in a 30 -min session with 60 escape trials induced by $0.3 \mathrm{~mA}$ footshocks delivered every $30 \mathrm{~s}$. Foot-shocks were terminated when the animal had a nose-poke, and lasted a maximum of $10 \mathrm{~s}$. For each trial, the green light was turned on during nonshock periods, and the red light was turned on during shock periods. The sensor located in the nose poke hole recorded the timing of each nose poke with $1 \mathrm{~s}$ resolution. Latency to escape and number of escapes were measured in blocks of five trials.

\section{Fixed Ratio Paradigm}

Water deprived mice were tested 5 days a week on the fixed ration paradigm and provided water access for $1 \mathrm{~h}$ per day. Each operant conditioning chamber was equipped as described above, but further contained a liquid dispenser fitted with a photocell sensor, situated in the middle of the wall left to the nose-poke module. Mice were habituated to the chamber for $30 \mathrm{~min}$, during which $10 \mu \mathrm{l}$ of water (=liquid reinforcer) were delivered to the liquid dipper every $30 \mathrm{~s}$. Mice were trained on a continuous fixed ratio (FR) schedule for which they had to nose-poke for the delivery of a liquid reinforcer during daily 30-min sessions across 12 days. Activation of the nose-poke led to delivery of $10 \mu \mathrm{l}$ water in the liquid dipper. Learning was determined by the ratio of total number of nose-pokes over total number of collected drink rewards.

\section{Environmental Enrichment}

Control and MSUS mice were placed in social groups $(n=12)$ in an enriched cage from weaning till adulthood. An enriched cage is a large box with two levels $(55 \times 36 \times 19 \mathrm{~cm}$ bottom level, $55 \times 36 \times 11 \mathrm{~cm}$ upper level $)$ (Marlau; Viewpoint). The bottom level is split into two compartments, one containing food pellets and the other providing access to water and containing running wheels, 
and a covered/protected area. The upper level has a maze $(35 \times 36 \times 11 \mathrm{~cm})$ whose shape and configuration are changed three times per week with a total of 12 different options. The box is organized such that animals have to go through the maze to reach the food compartment.

\section{Real-Time Quantitative RT-PCR}

DNaseI-treated RNA isolated from hippocampus (Allprep RNA/DNA kit; Qiagen) was reverse transcribed (RT) using the SuperScript First-Strand Synthesis System II for RT polymerase chain reaction (PCR; Invitrogen Carlsbad, California). Quantitative RT-PCR (qRT-PCR) was performed in an ABI 7500 thermal cycler using TaqMan probes (Applied Biosystems, Foster City, California; Mm00433832_m1) as described previously(Franklin et al, 2010).

\section{Bisulfite Pyrosequencing}

Genomic DNA from the hippocampus, prefrontal cortex, and sperm was extracted with the Allprep (Qiagen) and DNeasy blood and tissue (Qiagen) kit, respectively, according to the manufacturer's instructions. Bisulfite pyrosequencing of offspring hippocampus was performed by EpigenDx, USA. Universally methylated and unmethylated DNA samples (Millipore Bioscience Research Regents) were used as controls. Pyrosequencing of hippocampus, prefrontal cortex and sperm was performed as described previously (Bohacek et al, 2014). Amplicons containing the GR exon1-7 promoter region were generated using a standard PCR protocol, an unmodified forward primer (5'-GGTTTTGTAG GTTGGTTGTTATTT-3'), and a biotin-labeled reverse primer (5'-ATTTCTTTAATTTCTCTTCTCCCTAAC-3'). For highresolution sequencing, the following sequencing primer was used 5'-TTGTAGGTTGGTTGTTATTTTT-3'.

\section{Samples Preparation, iTRAQ Labeling, Mass Spectrometry Analyses and Protein Quantification}

Hippocampi dissected from adult mice were homogenized in $200 \mu \mathrm{l}$ lysis buffer $(100 \mathrm{mM}$ triethyl ammonium bicarbonate $\mathrm{pH} \sim 8,0.1 \%$ SDS, $2 \mathrm{M}$ urea) by up-and-down strokes using a 27 gauge syringe, sonicated for $2 \mathrm{~min}$, and centrifuged at $13000 \mathrm{~g}$ for $10 \mathrm{~min}$ to remove insoluble material. Proteins $(50 \mu \mathrm{g})$ were precipitated using six volumes ice-cold acetone, solubilized, reduced, and cysteines were blocked according to the manufacturer's protocol. Proteins were then digested into peptides with trypsin overnight (1:10 enzyme:substrate). Peptides were differentially labeled with iTRAQ 8-plex reagents (Applied Biosystems) according to the manufacturer's protocol then combined. Peptide samples were desalted and separated in-solution with an Agilent 3100 OFFGEL fractionator (Agilent Technologies) according to the manufacturer's protocol. Fractions were acidified by adding 50\% acetonitrile (ACN), 1\% trifluoroacetic acid (1:10), and detergents were removed using ZipTip C18 columns (Millipore). Samples were lyophilized then resuspended in 3\% ACN, $0.1 \%$ formic acid for LTQ-Orbitrap Velos analysis using collision-induced dissociation (CID), and higher energy collisional dissociation fragmentation as described in Uzozie et al (2014). In brief, for the eight most intense signals per cycle above a threshold of 1000, both CID and higher-energy collisional dissociation spectra were acquired in a data-dependent manner. Mascot Distiller 2.4.3.3 (Matrix Science, Boston, MA) was used to generate Mascot generic format peak lists. De-isotoping and peak picking were not performed between 112.5 and $121.5 \mathrm{~m} / z$ (range containing iTRAQ (isobaric tags for relative and absolute quantitation) reporter ions), and the CID and higher-energy collisional dissociation spectra were merged by summing. Peptide and protein identification was performed with ProteinPilot software v4.5 (AB Sciex) and the Paragon algorithm 4.5.0.0 (Shilov et al, 2007), by comparison with the mouse Swissprot/TrEMBL database (downloaded in May 2009). OGE fractions obtained by F1 analyses were searched separately from F2. Paragon method parameters were peptide labeled with iTRAQ 8plex, fixed modification of methyl methanethiosulfonate on Cys $(+46 \mathrm{Da})$, digestion with trypsin, instrument Orbi/FT MS (1-3 ppm) LTQ MS/MS and ID focus on biological modification with the thorough search effort. Proteins with at least one peptide above $90 \%$ confidence level as determined by Protein Pilot were recorded. For the estimation of protein abundance ratio, intensity of iTRAQ reporter ion areas for each non-shared, non-discordant, quantifiable, and confidently identified MS/MS spectra was exported from ProteinPilot. For each protein, the sum of each reporter channel was calculated across spectra matched to the same protein. Data were normalized for loading error by bias corrections using ProteinPilot. Statistical significance of differences between means for each group (Control and MSUS mice) was determined on the transformed protein sums (arc sin hyperbolic) using a two-tailed $t$-test. Fold changes were calculated using the group means of the protein sums. Proteins were considered regulated if the $P$ value was $<0.05$ and fold-change between groups $>1.3$-fold. Mass spectrometry proteomics data are deposited to the ProteomeXchange Consortium via the PRIDE [1] partner repository (identifier PXD004073).

\section{Statistical Analyses}

Two-way ANOVAs with treatment (Control and MSUS) and housing (standard and EE) as main factors were used to analyze data of light dark test, qRT-PCR, and bisulfite pyrosequencing. Repeated measurements ANOVAs with treatment and housing as main factors, and within factor of testing session (in blocks of 5 sessions), were used to analyze data of the active avoidance task. Repeated measurements ANOVAs with treatment as a main factor and testing session as within factor were used to analyze data of the FR paradigm. Two-tailed Student's $t$-tests were used to analyze qRT-PCR and bisulfite pyrosequencing data when comparing MSUS and control data in standard housing conditions only. All analyzed data matched the requirements for parametric statistical tests. If variance was not homogeneous between groups, adjusted $P$-value, $t$-value, and degree of freedom were reported. A value was considered outlier when deviating $>2$ SDs from the group mean, and outlier exclusion criterion was pre-established. Significance was set at $P<0.05$ for all tests. Error bars represent standard error of the mean (SEM). 


\section{a}

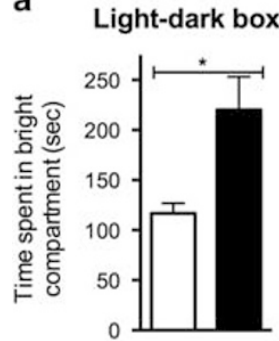

C

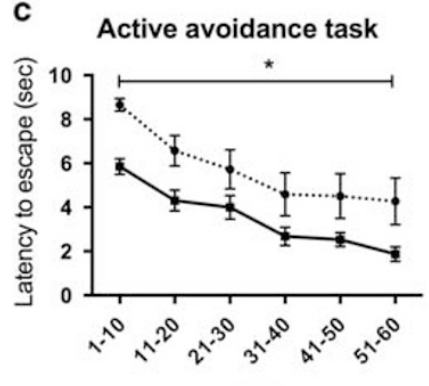

b

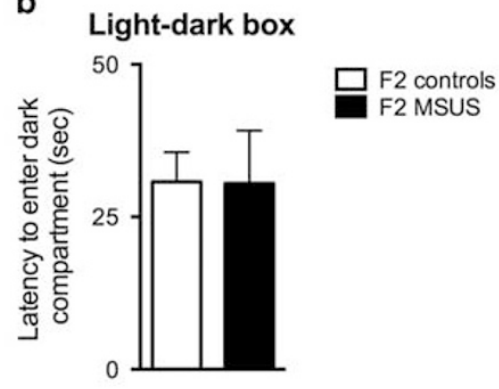

d

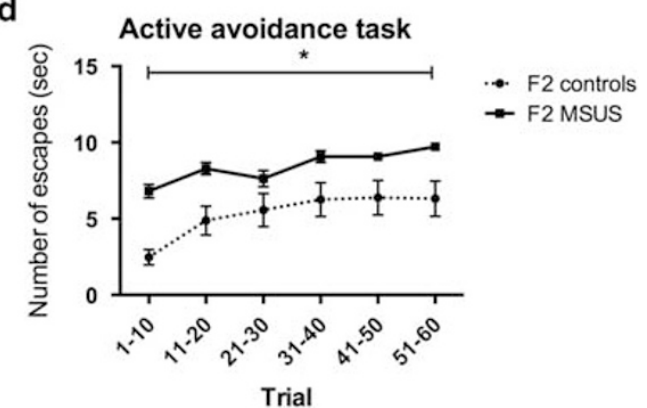

Figure I Aversive behavior in the offspring of males exposed to maternal separation combined with unpredictable maternal stress (MSUS) in postnatal life. Performance in a light-dark box shown by (a) time spent in the bright compartment in control and MSUS (Controls $n=33$, MSUS $n=36$ ) and (b) latency to first enter the dark compartment (Controls $n=34$, MSUS $n=38$ ). (c) The latency to escape a foot-shock and (d) the total number of foot-shocks on the active avoidance task (Controls $n=15$, MSUS $n=13$ ). Data are presented as mean \pm SEM, $* P<0.05$. Part of the data were published in Gapp et al. (20I4a).

\section{RESULTS}

\section{Effects of MSUS on Coping Behaviors in Aversive Conditions}

We used a light-dark box to assess behavioral response in mildly aversive conditions in males exposed to MSUS (F1) and their male offspring (F2) (Supplementary Figure S1). On this task, an animal can escape from a brightly lit aversive compartment of a box by moving to a dark compartment. F1 MSUS males spent more time in the bright compartment of the box than controls $(t(35)=-2.14, P<0.05$; Supplementary Figure S2a). Similarly, the F2 MSUS males also spent more time in the bright compartment $(t(41.61)=-3, P<0.01$; Figure 1a), indicating reduced escape response suggestive of a form of resistance to aversive conditions in both generations. This effect was independent from the latency to first enter the dark compartment since it was similar in all groups (fathers: $t(34)=0.023, P>0.05$, Supplementary Figure S2b; offspring, $t(63)=0.03, P>0.05$; Figure 1b). We confirmed this effect by testing the males in even more aversive conditions using an active avoidance task. On this task, an animal is exposed to a foot-shock that can be terminated by a nose-poke into a hole. F2 MSUS males had shorter latency to nose-poke $(\mathrm{F}(1,23)=8.50, P \leqslant 0.01)$ and thus received fewer foot-shocks than control offspring across time $(\mathrm{F}(1,26)=7.74, P \leqslant 0.01)$ (Figure $1 \mathrm{c}$ and $\mathrm{d}$ ), suggesting more active coping response. This was not due to improved learning since F2 MSUS and control males had similar nose-poke performance on a FR paradigm, a reward-based task for which a water-deprived animal learns to nose-poke into one hole to obtain a water reward in a neighboring hole $(\mathrm{F}(1,26)=1.33, P>0.05)$
(Supplementary Figure S3). These results suggest transmission of altered coping behaviors across generations.

\section{Reversibility of Altered Responses in Aversive Conditions}

EE has been reported to reverse some of the behavioral symptoms induced by chronic stress (Hutchinson et al, 2012) or early life adversity (Leshem and Schulkin, 2012). Using $\mathrm{EE}$, we examined if the effects of MSUS on coping behaviors can be reversed (Supplementary Figure S4). We exposed F1 MSUS males to EE from weaning till adulthood. After EE, F1 males were bred to control females to generate an F2 generation, and both F1 and F2 males were tested on the light-dark box. When compared with F1 control males raised in standard cages, F1 control males raised in EE spent more time in the lit compartment of the box $(t(52)=-0.9, P<0.05)$ and had shorter latency to enter the dark compartment $(t(52)=-2.11 ; P<0.05$, Supplementary Figure S2), suggesting an effect of EE on response to adverse conditions. F1 MSUS males exposed to EE had similar performance to control males exposed to EE; they spent a comparable amount of time in the lit compartment $(t(15)=-0.78, P>0.05)$ and had similar latency to first enter the dark compartment $(t(16)=-0.38), \quad P>0.05)$ (Supplementary Figure S2c and d). However, their F2 male offspring spent a similar amount of time in the lit compartment $(t(20.85)=1.94, P>0.05)$ and had lower latency $(t(17.89)=2.33, P<0.05)$ than the F2 offspring of control EE fathers (Figure 2a and $b$ ), suggesting a reversal of the altered response to an adverse environment by paternal EE. Decreased latency may indicate more spontaneous escape behavior in the F2 offspring of MSUS males exposed 
a
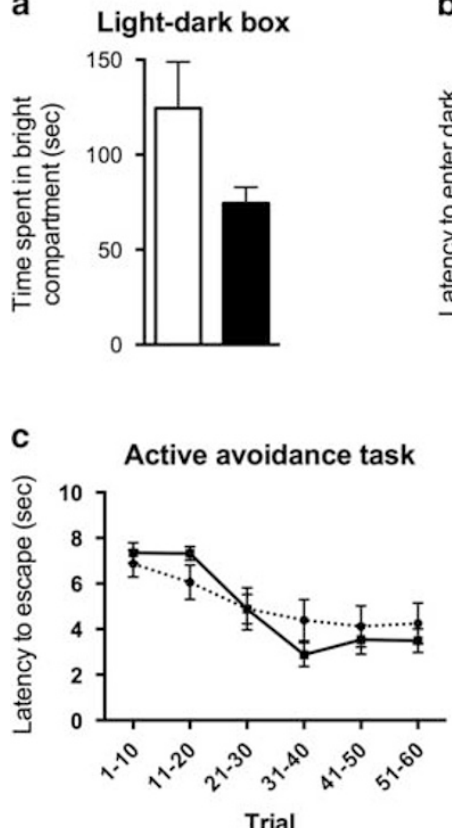

b

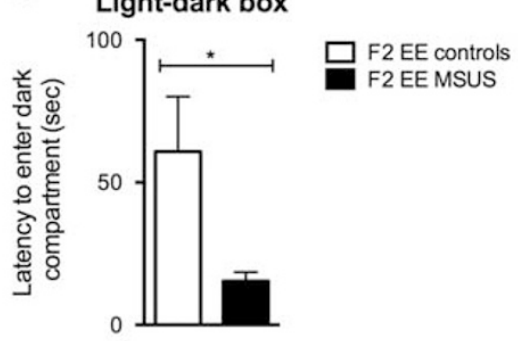

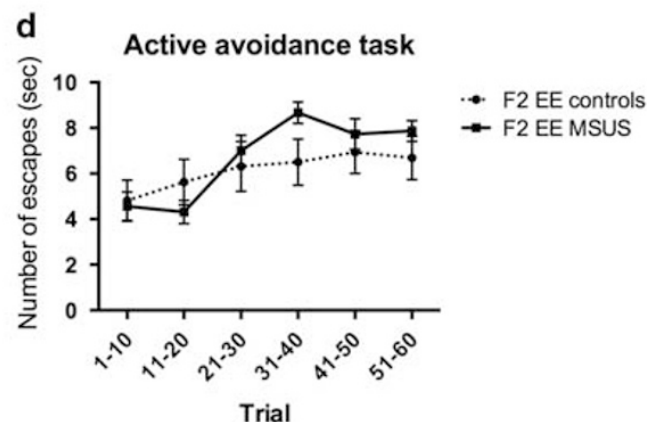

Figure 2 Aversive behavior in the offspring of males exposed to maternal separation combined with unpredictable maternal stress (MSUS) in postnatal life and environmental enrichment (EE) in adulthood. Performance in a light-dark box shown by (a) time spent in the bright compartment (EE: Controls $n=19$, MSUS $n=15$; group effect of housing $F(1,97)=6.09, P<0.05)$ ) and (b) latency to first enter the dark compartment (EE: Controls $n=18$, MSUS $n=16$; interaction between housing and treatment $F(1,97)=7.56, P<0.01$ )). (c) Latency to escape a foot-shock (group effect of treatment $F(I, 53)=4.72, P<0.05$; interaction between treatment and housing $F(I, 53)=3.07, P=0.09$ ) and $(d)$ total number of foot-shocks (group effect of treatment $F(I, 5 \mid)=7.22$, $P \leqslant 0.1$; interaction between treatment and housing $F(1,53)=3.41, P=0.07$ ) on the active avoidance task (EE: Controls $n=16, M S U S n=13$ ). Data are presented as mean \pm SEM. $* P<0.05$.

to EE. This is consistent with a decrease in the time spent in the bright compartment, suggesting a reduction in overall escape behavior in the F2 offspring of MSUS exposed to EE and a reversal of the effect of paternal MSUS exposed to standard housing. Similarly, responses on the active avoidance task were reversed by paternal EE in F2 MSUS males. The F2 offspring of MSUS fathers exposed to EE had similar latency to escape $(\mathrm{F}(1,27)=0.09, P>0.05)$ and number of escapes $(\mathrm{F}(1,28)=0.42, P>0.05)$ to the $\mathrm{F} 2$ offspring of control fathers exposed to EE (Figure $2 c$ and $d$ ).

\section{Effects of MSUS and EE on GR Expression and Promoter Methylation}

Since GR has been implicated in stress responses and coping behaviors, we examined if its expression is altered by MSUS in the hippocampus, a key brain structure implicated in the shut down of stress responses (de Kloet et al, 2005b). Using qRT-PCR, we observed a significant increase in GR expression in F2 MSUS males when compared with control males $(t(11)=-2.7, P<0.05)$. This increase was not observed in the offspring of F1 MSUS males exposed to EE $(t(12)=-0.84, P>0.05)$ (Figure 3a), indicating a correction of $G R$ expression by paternal EE. Hippocampal $G R$ expression was also increased in F1 MSUS fathers $(t(13)=-2.45, \quad P<0.05)$ and this increase was as well reversed by EE (Supplementary Figure S5). To assess the functional relevance of changes in GR expression, we conducted proteome-wide analyses of proteins in F1 and F2 hippocampus of control and MSUS males using a labeling method based on iTRAQ and mass spectrometry. This unbiased method allows the rigorous identification and quantification of multiple proteins at the same time in the same samples. The results of these proteomic analyses revealed that 17 proteins known to be glucocorticoidresponse element targets (Datson et al, 2011; Gray et al, 2014; Polman et al, 2012) were altered by at least 1.3 -fold in the hippocampus of adult MSUS males compared with adult control males. These changes seem to be a functional consequence of altered GR mRNA levels. They also significantly add to the functional relevance of the MSUS model by identifying the affected targets. The list of altered proteins and affected targets is provided in Table 1.

Because $G R$ gene is known to be regulated by DNA methylation (Weaver et al, 2004), we examined if DNA methylation is altered by MSUS. We used quantitative bisulfite pyrosequencing to measure DNA methylation in exons 1-7 of the GR gene, a region that contains transcription factor binding sites important for transcriptional regulation (Mueller and Bale, 2008; Figure 3c). In F2 MSUS offspring, exon 1-7 methylation was significantly decreased at CpG3 $(t(26)=2.33, P<0.05)$ and CpG7 $(t(26)=2.19, P<0.05)$ (Figure $3 b)$. Importantly, this decrease was reversed by paternal EE (CpG3: $t(10)=2.17, P>0.05$; CpG7: $t(10)=-0.97, P>0.05$; Figure $3 \mathrm{~b}$ ), indicating a correction of aberrant DNA methylation by EE in MSUS offspring. Since this effect accompanies a normalization of gene expression, it suggests functional relevance for $G R$ expression. Consistently in F1 MSUS males, DNA methylation at the $G R$ gene is lower and $G R$ expression is increased in the hippocampus (CpG2: $t(9)=3.43, P<0,01$; CpG3: $t(9)=2.31, P<0,05$; Supplementary Figure S6). We next 

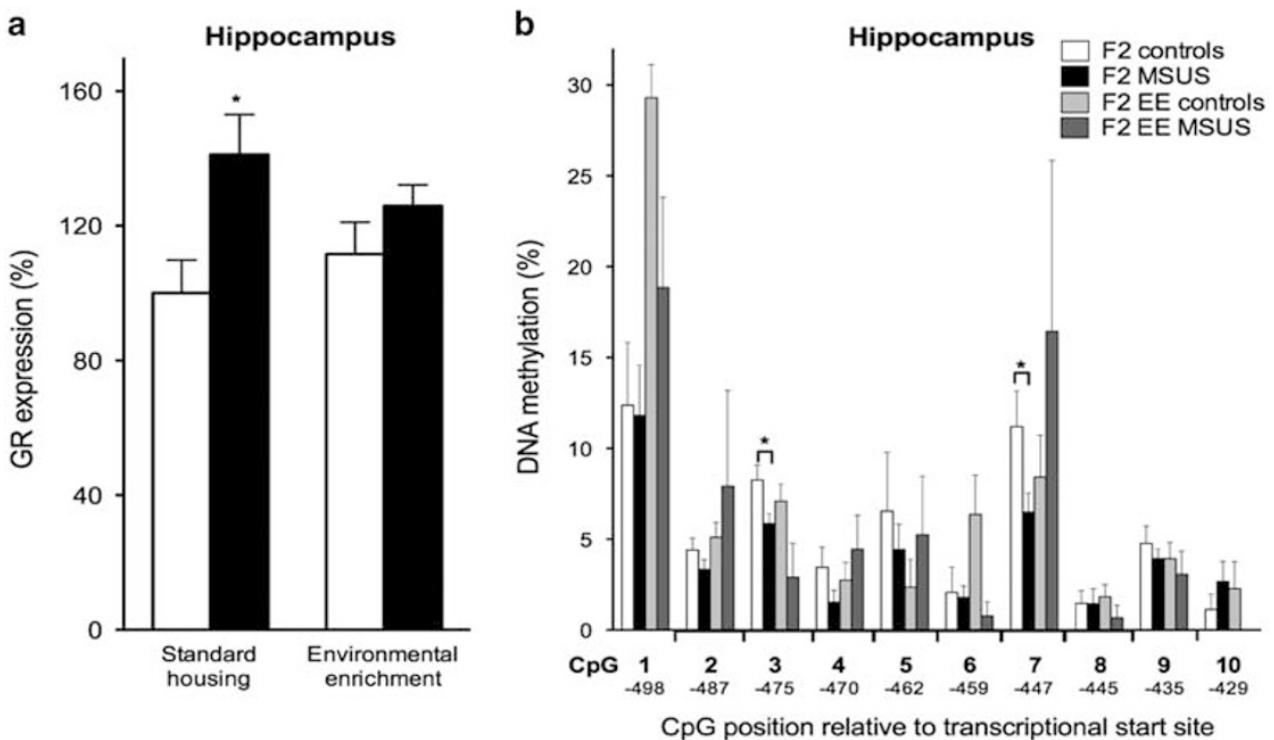

C

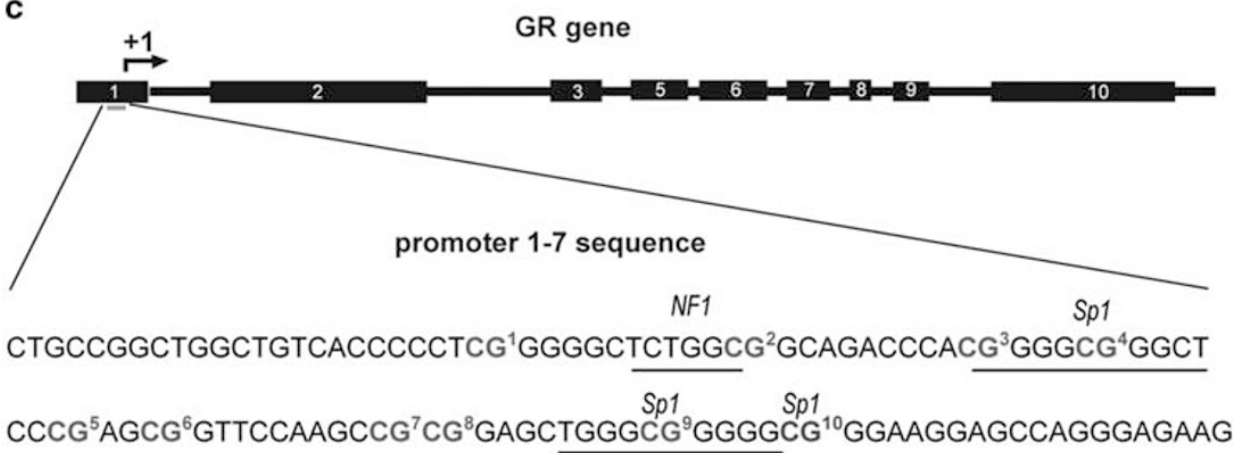

Figure 3 Molecular analysis in the F2 offspring of FI fathers exposed to maternal separation combined with unpredictable maternal stress (MSUS) and environmental enrichment (EE). (a) Glucocorticoid receptor (GR) gene expression measured by quantitative reverse transcriptase-PCR (Standard housing $(\mathrm{SH})$ : Controls $n=6$, MSUS $n=6$; EE: Controls $n=7$, MSUS $n=5$; group effect of treatment $F(I, 2 I)=7.74, P \leqslant 0.0 I$ ). (b) DNA methylation at GR exon I-7 promoter in the hippocampus assessed by bisulfite pyrosequencing (SH: Controls $n=14$, MSUS $n=14$; EE: Controls $n=7$, MSUS $n=5$; group effect of treatment at CpG2 $F(I, 20.8)=0.05$ and at $C p G 3: F(I, 6 I)=7.94, P \leqslant 0.01$; a tendency for group effect of housing at $C p G 3$ : $F(I, 23.31)=3.03$ and an interaction between housing and treatment at CpG4: $F(1 ; 43,9)=3.97, P \leqslant 0.05)$. (c) Schematic representation of the GR gene and the untranslated exon $I-7$ promoter sequence examined for DNA methylation by pyrosequencing assays including the transcription start site $(+I)$. Predicted transcription factor binding sites are underlined and in italic. Exons are shown as black boxes. Numbers represent individual CpG sites analyzed for DNA methylation (CpG I-I0). Data are presented as mean $\pm \mathrm{SEM}$, $* P<0.05$ t-test comparison.

examined if the altered DNA methylation is passed to the offspring from fathers by analyzing DNA methylation in sperm cells of F1 males. DNA methylation was significantly decreased at CpG3 $(t(19.4)=2.77, P<0.05, P<0.05)$ and CpG7 $(t(20)=2.3, P<0.05)$, consistent with hypomethylation at the same $\mathrm{CpG}$ in the offspring's hippocampus. We further detected a decrease at CpG1 $(t(14.03)=3.47$, $P<0.01), \quad$ CpG8 $\quad(t(14.07)=3.48, \quad P<0.01), \quad$ and $\quad$ CpG9 $(t(14.15)=3.5, P<0.01)$ (Figure 4). Importantly, these effects did not persist in the sperm of $\mathrm{F} 1$ fathers that had been exposed to EE, suggesting a correction of the effects of MSUS by EE at the germline level $(\mathrm{CpG} 1 t(8.03)=-19, P>0.05$, $P<0.05 ;$ CpG3 $t((8.02)=-2.09, P>0.05, P<0.05 ;$ CpG7 $t(9)=-2.26, \quad P=0.05, \quad$ CpG8 $\quad t((-8.01)=-2 ; \quad P>0.05$, $P>0.05$; CpG9 $t(8.03)=-2.12, P>0.05$; Figure 4$)$.

DNA methylation induced by MSUS in sperm may be maintained after fertilization and during development, and thus be present in different tissues in the offspring. We examined this question by assessing DNA methylation in another brain region, the prefrontal cortex, in adult 
Table I Proteome-Wide Protein Expression in the Hippocampus of FI MSUS Males and Their F2 Male Offspring

\begin{tabular}{|c|c|c|c|c|c|c|c|c|c|c|c|}
\hline Generation & $\begin{array}{l}\text { Protein accession } \\
\text { number }\end{array}$ & Gene name & Regulation & $\begin{array}{l}\text { Validated } \\
\text { GR target }\end{array}$ & \% Control & Generation & $\begin{array}{l}\text { Protein accession } \\
\text { number }\end{array}$ & Gene name & Regulation & $\begin{array}{l}\text { Validated } \\
\text { GR target }\end{array}$ & \% Control \\
\hline Fl fathers & Q69Z96 & Atp|3al & Down & & 420.0 & F2 offspring & O08908 & Pik3r2 & Down & & 63.6 \\
\hline Fl fathers & $|055| 42$ & Rpl35a & Down & & 65.9 & F2 offspring & | $055 \mid 42$ & Rpl35a & Down & & 42.8 \\
\hline FI fathers & O35343 & Kpna4 & Down & & 62.1 & F2 offspring & Q3UKW2 & Calml & Down & & 44.5 \\
\hline FI fathers & | Q9Z239 & Fxydl & Down & & 63.8 & F2 offspring & | Q9Z239 & Fxydl & Down & & 62.4 \\
\hline FI fathers & B9EКC3 & Arhgap5 & Down & & 61.0 & F2 offspring & Q62178 & Sema4a & Down & & 47.5 \\
\hline Fl fathers & P21995 & Emb & Down & & 64.9 & F2 offspring & Q7IM36 & Cspg5 & Down & & 63.5 \\
\hline Fl fathers & Q8JZK9 & HmgcsI & Down & & 70.4 & F2 offspring & P35802 & Gpm6a & Down & & 61.5 \\
\hline Fl fathers & Q6GQX8 & GmI5800 & Down & & 70.9 & F2 offspring & P55096 & Abcd3 & Down & & 66.2 \\
\hline Fl fathers & Q7TN99 & Cpeb3 & Down & & 73.6 & F2 offspring & Q8BGZ4 & $\mathrm{Cdc} 23$ & Down & & 60.1 \\
\hline Fl fathers & Q9JMG7 & Hdgfrp3 & Down & $x$ & 70.2 & F2 offspring & Q8K386 & Rab|5 & Down & & 60.7 \\
\hline FI fathers & P62137 & Ppplca & Down & & 76.2 & F2 offspring & Q9CQEI & Nipsnap3b & Down & & 57.0 \\
\hline Fl fathers & Q9QZB। & Rgs20 & Down & & 68.8 & F2 offspring & $\mathrm{A} 2 \mathrm{~B} 130$ & RP24-297HI7.3 & Down & & 64.2 \\
\hline Fl fathers & Q9CW46 & Raverl & Down & & 69.8 & F2 offspring & B9EKN8 & Tnik & Down & & 66.2 \\
\hline Fl fathers & Q9DI25 & Mrps25 & Down & & 74.2 & F2 offspring & Q9CPP6 & Ndufa5 & Down & & 56.1 \\
\hline Fl fathers & BIARB9 & $\mathrm{Ddx} 5$ & Down & & 76.2 & F2 offspring & Q9CZB4 & Apool & Down & $x$ & 30.4 \\
\hline FI fathers & Q9D6D0 & Slc25a27 & Down & & 76.8 & F2 offspring & Q8K356 & Ly6h & Down & & 58.2 \\
\hline Fl fathers & Q91W39 & Ncoa5 & Down & & 75.9 & F2 offspring & O70503 & Hsdl7b|2 & Down & & 58.4 \\
\hline Fl fathers & Q8VCE2 & GpnI & Down & & 72.5 & F2 offspring & Q3U0D9 & Hacel & Down & & 66.0 \\
\hline Fl fathers & Q9D6U8 & Fam I 62a & Down & & 75.6 & F2 offspring & Q81IS7 & Ubpl & Down & & 53.8 \\
\hline Fl fathers & Q8RIN4 & Nuded3 & Down & & 70.1 & F2 offspring & Q9ES97 & Rtn3 & Down & & 65.6 \\
\hline Fl fathers & Q9DC5I & Gnai3 & Down & & 72.4 & F2 offspring & Q9Z2W9 & Gria3 & Down & & 63.4 \\
\hline Fl fathers & Q52KF3 & Spirel & Up & $x$ & 165.8 & F2 offspring & Q78IK2 & Usmg5 & Down & & 60.4 \\
\hline Fl fathers & Q8CoW0 & Tmsb|5| & $U_{p}$ & & 154.7 & F2 offspring & Q62277 & Syp & Down & & 67.3 \\
\hline Fl fathers & PI4069 & S100a6 & $U_{p}$ & $x$ & 151.2 & F2 offspring & P52479 & Usp 10 & Down & $x$ & 71.0 \\
\hline $\mathrm{Fl}$ fathers & Q9D3D9 & Atp5d & Up & & 136.2 & F2 offspring & P56564 & Slcla3 & Down & & 72.8 \\
\hline Fl fathers & Q99LY9 & Ndufs5 & Up & & 132.3 & F2 offspring & Q9D9M2 & Usp 12 & Down & & 70.7 \\
\hline Fl fathers & Q99M87 & Dnaja3 & $U_{p}$ & $x$ & 148.6 & F2 offspring & Q9CR57 & Rpll 4 & Down & & 72.1 \\
\hline Fl fathers & POC7LO & Wipf3 & $U_{p}$ & $x$ & 136.3 & F2 offspring & P39688 & Fyn & Down & & 72.7 \\
\hline Fl fathers & B2RS2I & Nudt19 & Up & & 131.6 & F2 offspring & Q60930 & Vdac2 & Down & $x$ & 71.6 \\
\hline Fl fathers & P31786 & Dbi & $U_{p}$ & & 135.3 & F2 offspring & Q8CIP4 & Mark4 & Down & & 75.1 \\
\hline Fl fathers & Q3TY60 & Fam $\mid 3 / \mathrm{b}$ & Up & & 138.2 & F2 offspring & Q9CQN6 & Tmem I 4c & Down & & 73.7 \\
\hline Fl fathers & Q9נग $\mathrm{U} 8$ & Sh3bgrl & Up & & 131.8 & F2 offspring & Q3TCD4 & Eci2 & Down & & 69.8 \\
\hline Fl fathers & O08529 & Capn2 & $U_{p}$ & & 131.4 & F2 offspring & Q8BG32 & PsmdII & Down & $x$ & 74.0 \\
\hline Fl fathers & O35449 & Prrtl & Up & $x$ & 138.4 & F2 offspring & Q9ZIX2 & Ptdss2 & Down & & 66.8 \\
\hline Fl fathers & P62274 & Rps29 & Up & $x$ & 132.9 & F2 offspring & O70579 & Slc25al7 & Up & & 152.1 \\
\hline Fl fathers & P62996 & Tra2b & $U_{p}$ & & 140.3 & F2 offspring & Q2PFD7 & Psd3 & Up & & 154.1 \\
\hline Fl fathers & Q68ED7 & Crtcl & Up & & 142.6 & F2 offspring & Q8VHH5 & Agap3 & Up & & | 38.4 \\
\hline Fl fathers & Q8BK30 & Ndufv3 & Up & & 147.1 & F2 offspring & Q60972 & Rbbp4 & Up & & |38.1 \\
\hline Fl fathers & Q9DIQ6 & Erp44 & Up & & 133.0 & F2 offspring & Q8BGD9 & Eif4b & Up & & 131.1 \\
\hline Fl fathers & Q3U7M5 & Abhd4 & $U_{p}$ & & 142.1 & F2 offspring & BIAWD9 & Clta & $U_{p}$ & & 133.2 \\
\hline $\mathrm{Fl}$ fathers & Q9DBB8 & Dhdh & Up & & 141.7 & F2 offspring & Q9JLT4 & Txnrd2 & Up & & 141.5 \\
\hline $\mathrm{Fl}$ fathers & BIAQE6 & Ikzf3 & $U_{p}$ & & 144.0 & F2 offspring & Q7TN29 & Smap2 & Up & & 146.8 \\
\hline Fl fathers & Q3UH27 & Asap2 & Up & & 139.6 & F2 offspring & Q52KR3 & Prune2 & Up & & 133.5 \\
\hline Fl fathers & P16390 & Kcna3 & $U_{p}$ & $x$ & 131.7 & F2 offspring & Q61166 & Maprel & Up & & 144.9 \\
\hline \multirow[t]{3}{*}{ FI fathers } & Q52KR3 & Prune2 & $U_{p}$ & & 133.3 & F2 offspring & Q6R891 & Ppplr9b & $U_{p}$ & & 130.5 \\
\hline & & & & & & F2 offspring & A2AN47 & Golga2 & Up & & 131.7 \\
\hline & & & & & & F2 offspring & P97930 & Dtymk & Up & & 140.0 \\
\hline
\end{tabular}

Proteins with differential expression $(P<0.05)$ in MSUS and controls and a minimal fold change of 1.3 measured by iTRAQ followed by mass spectrometry. Proteins with significanlty different level $(p \leqslant 0.05)$ of at least I.3-fold. Proteins significantly dysregulated in both $\mathrm{FI}$ and $\mathrm{F} 2$ generations are in grey tint. 


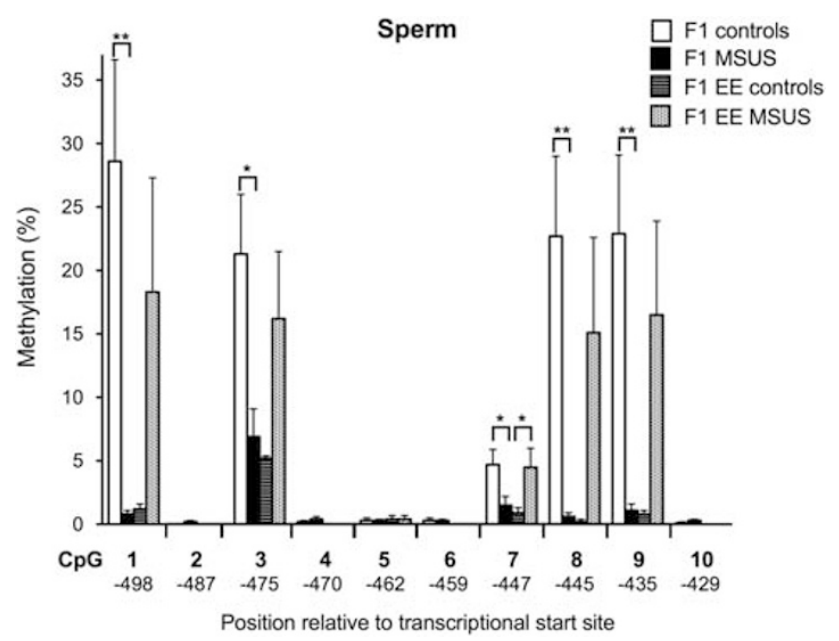

Figure 4 DNA methylation in sperm from males exposed to matemal separation combined with unpredictable maternal stress (MSUS) and environmental enrichment (EE). GR exon 1-7 promoter methylation in sperm cells of fathers assessed by bisulfite pyrosequencing ( $\mathrm{SH}$ : Controls $n=15$, MSUS $n=10$; EE: Controls $n=11, \operatorname{MSUS} n=9$; interaction between treatment and housing at CpG| $F(|, 6043.7|)=\mid 3.19, P<0.01 ; C p G 3$ $F(1,0.39)=14, \quad P<0.01 ; \quad C p G 7 \quad F(|| 73.42)=14.18,, \quad P<0.01 ; \quad C p G 8$ $F(\mid, 3832.58)=|3.59, P<0.01 ; C p G 9 F(\mid, 3900.57)=| 4.28, P<0.0 \mid)$. Data are presented as mean $\pm \mathrm{SEM}, * P \leqslant 0.05$, $* * * \leqslant 0.01$, $t$-test comparison.

offspring. Bisulfite pyrosequencing analyses in prefrontal cortex revealed that DNA methylation was not altered at CpG3 $(t(14)=-0.17$; Supplementary Figure S7). Instead, DNA methylation was decreased at CpG2 $(t(13.77)=2.64$, $P<0.05, P<0.05)$ and CpG7 $(t(14)=1.94 ; P<0.1)$ in the F2 offspring of MSUS males exposed to standard housing (Supplementary Figure S7). These effects were not observed in the F2 offspring of MSUS males exposed to EE at CpG2 $(t(13)=-1.01 ; \quad P>0.05)$, and were reversed at CpG7 $(t(13)=-2.26 ; P<0.05)$ (Supplementary Figure S7). Further, MSUS increased DNA methylation in the prefrontal cortex at CpG4, in both the F2 offspring of standard housed and EE fathers (Supplementary Figure S7). The observed changes differ from those in the hippocampus, suggesting that changes in DNA methylation in sperm cells are not systematically passed to all cells in the offspring. Region/ tissue-specific mechanisms likely mediate the transgenerational effects and the interplay of early life stress and housing conditions.

\section{DISCUSSION}

The present results show that the offspring of males exposed to traumatic stress during early postnatal life are better able to appraise and respond to adversity when adult. At a molecular level, this effect is associated with GR and involves increased $G R$ expression and decreased DNA methylation of $G R$ promoter in the hippocampus. Strikingly, the behavioral changes are reversible and behavior in the offspring is normalized when fathers are exposed to EE in adulthood. This is accompanied by a correction of DNA hypomethylation at some CpGs of the GR gene in the sperm of fathers and the hippocampus of the offspring.
In humans and primates, traumatic experiences often increase the risk for psychopathology but in some conditions, they can lead to resilience (Lyons and Parker, 2007), a form of active coping when faced with a challenging environment, later in life (Zozulya et al, 2008). Similarly in rodents, successful adaptation after exposure to chronic stress in early life has been observed, and has been associated with changes in the HPA axis (Franklin et al, 2011; Gapp et al, 2014b; Uchida et al, 2011). Thus, the response of individuals exposed to negative experiences in early life can be pathological or adaptive depending on the context, a concept theorized by the match/mismatch hypothesis (Daskalakis et al, 2012). The present results are in line with this hypothesis because they show that the offspring of males exposed to early trauma can cope better with a challenging situation, similarly to that experienced by their father. Further, they extend previous observations that MSUS animals have improved behavioral flexibility in aversive conditions (Gapp et al, 2014b), suggesting that exposure to early trauma can lead to various forms of resilience.

Active avoidance is a form of adaptive behavior that involves, in part, $G R$ expression in the hippocampus (Korte, 2001). One of the known modes of regulation of GR expression implicates DNA methylation at exons 1-7 of the GR gene. GR expression is known to be modulated by maternal care (Weaver et al, 2004). Here, we show that GR expression is also responsive to other factors, in particular traumatic stress in postnatal life and EE in adulthood. GR expression has been reported to be sex-specific at baseline (Elakovic et al, 2011) and has been linked to sex-specific responses in changing environments (Lin et al, 2011). Interestingly, while GR expression is increased in MSUS males, it is not altered in MSUS females (Gapp et al, 2014b), indicating sex-specific alteration by traumatic stress. The mechanisms for male-specific effects are not known but could involve DNA methylation. For instance, several algorithms predict the existence of a binding site for the transcription factor SP1 at CpG3, a CpG affected by MSUS (Tsunoda and Takagi, 1999). SP1 is known to regulate GR expression in humans (Suehiro et al, 2004) and other mammals (Zou et al, 2013), and act by dimerizing with the sex hormone receptor ER (Klinge, 2001). SP1-ER interactions could differentially interfere with the hippocampal response to stress causing sex-specific differences in expression.

Our data on DNA methylation in F1 sperm and F2 hippocampus suggest the implication of this epigenetic mark in the regulation of $G R$ gene expression and the inheritance of its alterations in our model. At some CpGs, DNA methylation was reduced in the sperm of F1 fathers and the hippocampus of their offspring. The alterations were corrected in sperm by paternal EE and DNA methylation was normal at these sites in the offspring. However, at this stage, it is not possible to determine whether and which CpG(s) directly control the regulation of GR expression, and it is likely that other mechanisms are also implicated. Our proteomic data indeed suggest that several proteins involved in gene transcription, protein translation, peptide and amide biosynthesis relevant to $G R$ expression may also contribute since they were differentially expressed in the hippocampus of F1 or F2 MSUS males compared with controls. In particular, the nuclear receptor coactivator 5 (Ncoa5), 
a putative binding partner of steroid receptors like GR that can act as a transcriptional coactivator or repressor, was found to be downregulated. Further, the human growth and transformation-dependent protein (HGTDP or Fam162a), involved in GR localization upon activation to the nucleus, are downregulated. In contrast, DNAJ3a a direct interaction partner of GR (Hedman et al, 2006) is upregulated. DNAJ3a stimulates Hsp70 leading to GR inactivation (Kirschke et al, 2014) and impacting GR autoregulation. These results therefore suggest that, in the brain, GR expression is likely controlled by different mechanisms that may complement DNA methylation.

$\mathrm{EE}$ is a non-invasive and natural paradigm that increases the level of sensory, cognitive, and motor stimulation and promotes brain plasticity. It has been used to restore hippocampal integrity after chronic stress (Hutchinson et $a l, 2012$ ) and may have some potential to counteract symptoms of psychopathologies and neurodegenerative diseases (Hannan, 2014; Nithianantharajah and Hannan, 2006). Our findings demonstrate that EE can reverse the effects of early traumatic stress. The reversal might involve adaptive mechanisms resulting from the mismatch between favorable EE conditions and adverse conditions in early life. These mechanisms may also involve epigenetic regulation of GR gene in the hippocampus, consistent with the idea that chronic stress and EE recruit similar pathways within the hippocampus (Hutchinson et al, 2012). Additional molecular pathways may also be involved. Besides regulating GR via DNA methylation, EE might regulate GR at the transcriptional level. Since GR is an auto- and cross-regulated gene, initial hypermethylation observed in the sperm of environmentally enriched MSUS males might first lead to decreased GR expression. This might release the negative autofeedback by GR and thereby normalize GR levels while at the same time, affect the recruitment of epigenetic enzymes such as DNA methyl transferases/demethylases that ultimately decrease DNA methylation.

The mechanisms underlying germline-dependent epigenetic inheritance remain not fully understood but have been proposed to implicate one or more epigenetic marks including DNA methylation, histone/protamine posttranslational modifications and RNA (Gapp et al, 2014c). Here, we show that DNA methylation likely contributes to the transmission of alterations in GR expression in response to environmental exposures such as MSUS and EE. This suggests that DNA methylation is modifiable at some CpGs during postnatal life and adulthood. The data also show that while some changes in sperm methylome are present in the offspring, some are not maintained in the offspring, suggesting corrective mechanisms. These mechanisms are likely tissue- and cell-specific. The causal involvement of DNA methylation in epigenetic inheritance remains however to be demonstrated. But this is complicated by the large inter-individual variability of the methylome in sperm, which is a strong confounding factor (Laurentino et al, 2016). Our previous work demonstrated the involvement of sperm RNA in the transmission of some behavioral and metabolic traits in the MSUS model (Gapp et al, 2014a). Sperm non-coding RNAs like microRNAs could also be involved in the transgenerational regulation of $G R$ gene but to date, no miRNA that can target GR have been identified in MSUS sperm (Gapp et al, 2014a).
In summary, our data provide new evidence that some forms of coping can be induced by trauma exposure in early life and be transmitted to the offspring. They also newly demonstrate that behavior and the epigenome in the germline and the brain are sensitive to the environment and can be modified across life. These findings have potential implications for the design of diagnostic and therapeutic approaches for trauma-related psychopathologies.

\section{FUNDING AND DISCLOSURE}

The Mansuy laboratory is supported by the University of Zürich, the Swiss Federal Institute of Technology, the Swiss National Science Foundation, Roche. Katharina Gapp was supported by a fFORTE fellowship by the Austrian Academy of Science. The authors declare no conflict of interest.

\section{ACKNOWLEDGMENTS}

We thank Tamara Franklin for help with samples preparation, Megan Frugoli for help with behavioral testing, animal care takers for their excellent work, and Carmen Sandi for critical discussion of the results. KG, JB, and FM conducted MSUS and EE paradigms and collected tissue. KG and FM performed behavioral testing. KG conducted real-time qRT-PCR. KG and JB performed pyrosequencing experiments. AMB carried out ITRAQ labeling and mass spectrometry measurements with assistance of PN. Proteomic data were analyzed by JG. KG and IMM designed the experiments, interpreted the results, and wrote the manuscript.

\section{REFERENCES}

Bohacek J, Farinelli M, Mirante O, Steiner G, Gapp K, Coiret G et al (2014). Pathological brain plasticity and cognition in the offspring of males subjected to postnatal traumatic stress. Mol Psychiatry 20: $621-631$.

Bohacek J, Mansuy IM (2015). Molecular insights into transgenerational non-genetic inheritance of acquired behaviours. Nat Rev Genet 16: 641-652.

Daskalakis NP, Oitzl MS, Schachinger H, Champagne DL, de Kloet ER (2012). Testing the cumulative stress and mismatch hypotheses of psychopathology in a rat model of early-life adversity. Physiol Behav 106: 707-721.

Datson NA, Polman JA, de Jonge RT, van Boheemen PT, van Maanen EM, Welten J et al (2011). Specific regulatory motifs predict glucocorticoid responsiveness of hippocampal gene expression. Endocrinology 152: 3749-3757.

de Kloet ER, Joels M, Holsboer F (2005a). Stress and the brain: from adaptation to disease. Nat Rev Neurosci 6: 463-475.

de Kloet ER, Sibug RM, Helmerhorst FM, Schmidt MV (2005b). Stress, genes and the mechanism of programming the brain for later life. Neurosci Biobehav Rev 29: 271-281.

Elakovic I, Djordjevic A, Adzic M, Djordjevic J, Radojcic M, Matic G (2011). Gender-specific response of brain corticosteroid receptors to stress and fluoxetine. Brain Res 1384: 61-68.

Franklin TB, Linder N, Russig H, Thony B, Mansuy IM (2011). Influence of early stress on social abilities and serotonergic functions across generations in mice. PLoS One 6: e21842.

Franklin TB, Russig H, Weiss IC, Graff J, Linder N, Michalon A et al (2010). Epigenetic transmission of the impact of early stress across generations. Biol Psychiatry 68: 408-415. 
Franklin TB, Saab BJ, Mansuy IM (2012). Neural mechanisms of stress resilience and vulnerability. Neuron 75: 747-761.

Gapp K, Jawaid A, Sarkies P, Bohacek J, Pelczar P, Prados J et al (2014a). Implication of sperm RNAs in transgenerational inheritance of the effects of early trauma in mice. Nat Neurosci 17: 667-669.

Gapp K, Soldado-Magraner S, Alvarez-Sanchez M, Bohacek J, Vernaz G, Shu H et al (2014b). Early life stress in fathers improves behavioural flexibility in their offspring. Nat Commun 5: 5466.

Gapp K, von Ziegler L, Tweedie-Cullen RY, Mansuy IM (2014c). Early life epigenetic programming and transmission of stressinduced traits in mammals: how and when can environmental factors influence traits and their transgenerational inheritance? BioEssays 36: 491-502.

Gray JD, Rubin TG, Hunter RG, McEwen BS (2014). Hippocampal gene expression changes underlying stress sensitization and recovery. Mol Psychiatry 19: 1171-1178.

Hannan AJ (2014). Environmental enrichment and brain repair: harnessing the therapeutic effects of cognitive stimulation and physical activity to enhance experience-dependent plasticity. Neuropathol Appl Neurobiol 40: 13-25.

Hedman E, Widen C, Asadi A, Dinnetz I, Schroder WP, Gustafsson JA et al (2006). Proteomic identification of glucocorticoid receptor interacting proteins. Proteomics 6: 3114-3126.

Hutchinson KM, McLaughlin KJ, Wright RL, Bryce Ortiz J, Anouti DP, Mika A et al (2012). Environmental enrichment protects against the effects of chronic stress on cognitive and morphological measures of hippocampal integrity. Neurobiol Learn Mem 97: 250-260.

Jankord R, Herman JP (2008). Limbic regulation of hypothalamopituitary-adrenocortical function during acute and chronic stress. Ann NY Acad Sci 1148: 64-73.

Kirschke E, Goswami D, Southworth D, Griffin PR, Agard DA (2014). Glucocorticoid receptor function regulated by coordinated action of the Hsp90 and Hsp70 chaperone cycles. Cell 157: 1685-1697.

Klengel T, Binder EB (2015). Epigenetics of stress-related psychiatric disorders and gene x environment interactions. Neuron 86: 1343-1357.

Klinge CM (2001). Estrogen receptor interaction with estrogen response elements. Nucleic Acids Res 29: 2905-2919.

Korte SM (2001). Corticosteroids in relation to fear, anxiety and psychopathology. Neurosci Biobehav Rev 25: 117-142.

Kovalenko IL, Galyamina AG, Smagin DA, Michurina TV, Kudryavtseva NN, Enikolopov G (2014). Extended effect of chronic social defeat stress in childhood on behaviors in adulthood. PLoS One 9: e91762.

Laurentino S, Borgmann J, Gromoll J (2016). On the origin of sperm epigenetic heterogeneity. Reproduction 151: R71-R78.

Leshem M, Schulkin J (2012). Transgenerational effects of infantile adversity and enrichment in male and female rats. Dev Psychobiol 54: $169-186$

Lin EJ, Choi E, Liu X, Martin A, During MJ (2011). Environmental enrichment exerts sex-specific effects on emotionality in C57BL/ 6J mice. Behav Brain Res 216: 349-357.

Liu D, Diorio J, Tannenbaum B, Caldji C, Francis D, Freedman A et al (1997). Maternal care, hippocampal glucocorticoid receptors, and hypothalamic-pituitary-adrenal responses to stress. Science 277: $1659-1662$.
Lyons DM, Parker KJ (2007). Stress inoculation-induced indications of resilience in monkeys. J TraumaStress 20: 423-433.

Metz GA, Ng JW, Kovalchuk I, Olson DM (2015). Ancestral experience as a game changer in stress vulnerability and disease outcomes. BioEssays 37: 602-611.

Mueller BR, Bale TL (2008). Sex-specific programming of offspring emotionality after stress early in pregnancy. $J$ Neurosci 28: 9055-9065.

Nithianantharajah J, Hannan AJ (2006). Enriched environments, experience-dependent plasticity and disorders of the nervous system. Nat Rev Neurosci 7: 697-709.

Patacchioli FR, Casolini P, Puglisi-Allegra S, Sadile AG, Angelucci L (1990). Hippocampal glucocorticoid receptor and behavior: a correlative study in rats and mice. J Steroid Biochem Mol Biol 37: 405-409.

Plotsky PM, Meaney MJ (1993). Early, postnatal experience alters hypothalamic corticotropin-releasing factor (CRF) mRNA, median eminence CRF content and stress-induced release in adult rats. Brain Res Mol Brain Res 18: 195-200.

Polman JA, Welten JE, Bosch DS, de Jonge RT, Balog J, van der Maarel SM et al (2012). A genome-wide signature of glucocorticoid receptor binding in neuronal PC12 cells. BMC Neurosci 13: 118.

Schloesser RJ, Lehmann M, Martinowich K, Manji HK, Herkenham M (2010). Environmental enrichment requires adult neurogenesis to facilitate the recovery from psychosocial stress. Mol Psychiatry 15: 1152-1163.

Shilov IV, Seymour SL, Patel AA, Loboda A, Tang WH, Keating SP et al (2007). The Paragon Algorithm, a next generation search engine that uses sequence temperature values and feature probabilities to identify peptides from tandem mass spectra. Mol Cell Proteomics 6: 1638-1655.

Suehiro T, Kaneda T, Ikeda Y, Arii K, Kumon Y, Hashimoto K (2004). Regulation of human glucocorticoid receptor gene transcription by $\mathrm{Sp} 1$ and p53. Mol Cell Endocrinol 222: 33-40.

Tsunoda T, Takagi $\mathrm{T}$ (1999). Estimating transcription factor bindability on DNA. Bioinformatics 15: 622-630.

Uchida S, Hara K, Kobayashi A, Otsuki K, Yamagata H, Hobara T et al (2011). Epigenetic status of Gdnf in the ventral striatum determines susceptibility and adaptation to daily stressful events. Neuron 69: 359-372.

Uzozie A, Nanni P, Staiano T, Grossmann J, Barkow-Oesterreicher S, Shay JW et al (2014). Sorbitol dehydrogenase overexpression and other aspects of dysregulated protein expression in human precancerous colorectal neoplasms: a quantitative proteomics study. Mol Cell Proteomics 13: 1198-1218.

Weaver IC, Cervoni N, Champagne FA, D'Alessio AC, Sharma S, Seckl JR et al (2004). Epigenetic programming by maternal behavior. Nat Neurosci 7: 847-854.

Zou H, Jiang Z, Li R, Jia Y, Yang X, Ni Y et al (2013). p53 cooperates with $\mathrm{Sp} 1$ to regulate breed-dependent expression of glucocorticoid receptor in the liver of preweaning piglets. PLoS One 8: e70494.

Zozulya AA, Gabaeva MV, Sokolov OY, Surkina ID, Kost NV (2008). Personality, coping style, and constitutional neuroimmunology. J Immunotoxicol 5: 221-225.

Supplementary Information accompanies the paper on the Neuropsychopharmacology website (http://www.nature.com/npp) 